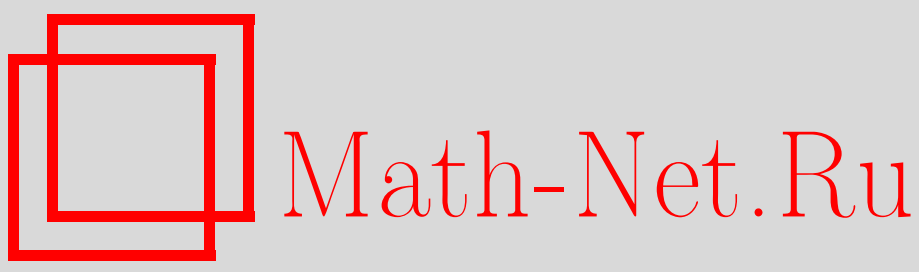

М. И. Анохин, О базисе тождеств многообразия, порожденного конечно базируемым квазимногообразием групп, Матем. сб., 1998, том 189, номер 8, 3-12

DOI: https://doi.org/10.4213/sm330

Использование Общероссийского математического портала Math-Net.Ru подразумевает, что вы прочитали и согласны с пользовательским соглашением http://www . mathnet.ru/rus/agreement

Параметры загрузки:

IP: 35.173 .219 .149

26 апреля 2023 г., 17:05:06 
УДК 512.54.01

\author{
М.И. Анохин
}

\title{
О базисе тождеств многообразия, порожденного конечно базируемым квазимногообразием групп
}

Построены примеры разрешимых (в том числе ступени не более 7) конечно базируемых квазимногообразий групп, которые порождают не конечно базируемые многообразия.

Библиографиия: 6 названий.

\section{Введение и формулировка основного результата}

В настоящей работе будет доказана

ТЕОРема 1. Существуют разрешимые (в том числе ступени не более 7) конечно базируемые квазимногообразия групп, которые порожсдают не конечно базируемые многообразия.

Это дает отрицательный ответ на вопрос А. В. Кузнецова [1, вопрос 8.28]. Явное описание примеров квазимногообразий, удовлетворяющих условию теоремы 1 , приведено в начале $\S 4$.

Основным методом работы является метод, развитый Ю.Г. Клейманом в [2], которьй, в свою очередь, восходит к идеям работ [3], [4]. Этот метод позволяет сводить изучение некоторых подгрупп некоторой относительно свободной группы к изучению подмножеств в более просто устроенных (например, нильпотентных) относительно свободных группах.

\section{§1. Определения, обозначения и необходимые факты}

Пусть $F=F_{\infty}-$ свободная группа счетного ранга. Зафиксируем алфавит $t_{1}, t_{2}, \ldots$ в качестве системы свободных порождающих $F$ и будем называть элементы этой группы словами от $t_{1}, t_{2}, \ldots$. Для множества $M$ символ $M^{*}$ обозначает множество всех бесконечных последовательностей $m_{1}, m_{2}, \ldots$ элементов $M$. Буквы $t_{i}$ можно рассматривать как переменные, тогда значением слова $v(t)=v\left(t_{1}, t_{2}, \ldots\right)$ в произвольной групше $G$ называется любой элемент вида $v(g)=v\left(g_{1}, g_{2}, \ldots\right)$, где $g=g_{1}, g_{2}, \ldots \in G^{*}$ (разумеется, $v(g)$ зависит лишь от некоторой конечной подпоследовательности последовательности $g$ ). Множество всех значений слова $v(t)$ в групше $G$ мы обозначим через $v[G]$, а соответствуюшую вербальную подгруппу, т.е. $\langle v[G]\rangle$, через $v(G)$ ( $\langle M\rangle$ обозначает группу, порожденную множеством $M)$. Вербальная подгруппа групшы $G$, соответствующая многообразию групп $\mathfrak{V}$, обозначается через $\mathfrak{V}(G)$.

Под $\mathfrak{E}, \mathfrak{O}, \mathfrak{A}, \mathfrak{N}_{c}, \mathfrak{B}_{e}, \mathfrak{A}_{e}$ понимается, соответственно, единичное многообразие, многообразие всех груп, многообразие всех абелевых групп, многообразие

$$
\text { (C) М.и. Анохин } 1998
$$


всех нильпотентных групп класса не более $c$, бернсайдово многообразие экспоненты $e$ (т.е. многообразие, определенное тождеством $t_{1}^{e}=1$ ) и многообразие $\mathfrak{A} \cap \mathfrak{B}_{e}$. Пусть также $\mathbb{Z}$ - кольцо целых чисел, $\mathbb{Z}_{2}=\mathbb{Z} / 2 \mathbb{Z}$ - поле из двух элементов, $\mathbb{N}_{k}=\{n \in \mathbb{Z}: n \geqslant k\}, R G$ - групповое кольцо группы $G$ над кольцом $R, G=G_{(1)} \supseteq G_{(2)} \supseteq \cdots-$ нижний центральньй ряд группы $G, G^{\prime}=G_{(2)}$ - коммутант группы $G, G^{e}=\mathfrak{B}_{e}(G)$ - подгруппа, порожденная $e$-ми степенями всевозможных элементов $G, a^{b}=b^{-1} a b,[a, b]=a^{-1} b^{-1} a b$ (коммутатор $a$ и $b$ ), $[M, N]=\{[x, y]: x \in M, y \in N\}$, где $a, b$ - элементы, а $M, N$-подмножества произвольной группы. Коммутатор вида $\left[a_{1}, a_{2}, a_{3}, \ldots, a_{n}\right]$ предполагается левонормированным, т.е. равным $\left[\ldots\left[\left[a_{1}, a_{2}\right], a_{3}\right], \ldots, a_{n}\right]$. Для удобства вхождение в коммутатор подряд $n$ одинаковых элементов $a$ мы будем записывать через $n a$, т.е.

$$
[\ldots, \underbrace{a, \ldots, a}_{n \text { раз }}, \ldots]=[\ldots, n a, \ldots] .
$$

Напомним, что для многообразий групा $\mathfrak{U}$ и $\mathfrak{V}$ через [U, $\mathfrak{V}]$ обозначается многообразие, определенное множеством тождеств $\{w(t)=1: w(t) \in[\mathfrak{U}(F), \mathfrak{V}(F)]\}$ (см. также [5, гл. $2, \S 1])$.

Пусть $\mathfrak{C}$ - некоторый класс групп. Через var $\mathfrak{C}$ будет обозначаться многообразие, порожденное классом $\mathfrak{C}$. Для произвольной формулы $\Phi$ исчисления предикатов в групповой сигнатуре запись $\mathfrak{C} \models \Phi$ будет означать, что формула $\Phi$ тождественно истинна в классе $\mathfrak{C}$. Если класс $\mathfrak{C}$ состоит из одной группы $G$, то вместо $\{G\} \models \Phi$ мы будем писать $G \models \Phi$.

Следуя [2], определим следуюшие объекты: $\mathfrak{R}$ - произвольное многообразие, отличное от $\mathfrak{E}$ и $\mathfrak{O} ; \mathfrak{L}=\mathfrak{A}_{2}^{3} \cap\left[\mathfrak{A}_{2}^{2}, \mathfrak{E}\right] ; R=F_{\infty}(\mathfrak{R}), A=F_{\infty}\left(\mathfrak{A}_{2} \mathfrak{R}\right), S=F_{\infty}(\mathfrak{L} \mathfrak{R})$, $H=F_{\infty}\left(\mathfrak{A}_{2}\right)$ - свободные группы счетного ранга соответствующих многообразий; $\left\{x_{1}, x_{2}, \ldots\right\},\left\{y_{1}, y_{2}, \ldots\right\},\left\{s_{1}, s_{2}, \ldots\right\},\left\{h_{1}, h_{2}, \ldots\right\}$ - системы свободных порождаюших $R, A, S, H$, соответственно. Пусть также $L=\mathfrak{R}(S)$, тогда легко видеть, что $L$ - свободная группа счетного ранга многообразия $\mathfrak{L}$ и $L^{4}$ - элементарная абелева 2-группа, содержашаяся в центре $L$.

ЛЕмма 1.1 ([2, лемма 4.1]). 1) Пусть $U$ - множество представителей всех смежных классов $L \backslash L^{2}$ по $L^{2}$. Тогда множество $\left\{u^{4}: u \in U\right\}$ является базисом әлементарной абелевой 2-группь $L^{4}$.

2) Пусть $v_{1}(t), v_{2}(t) \in \mathfrak{R}(F)$. Тогда

$$
v_{1}^{4}(s)=v_{2}^{4}(s) \Longleftrightarrow v_{1}(y)=v_{2}(y) .
$$

Очевидно, что ядром естественного эпиморфизма $S \rightarrow A$ (т.е. эпиморфизма, отображающего $s_{i}$ в $y_{i}$ для всех $\left.i \in \mathbb{N}_{1}\right)$ является $L^{2}$. Поэтому если $v_{1}(s) \equiv v_{2}(s)$ $\left(\bmod L^{2}\right)$, где $v_{1}(t), v_{2}(t) \in \mathfrak{R}(F)$, то $v_{1}(y)=v_{2}(y)$ и $v_{1}^{4}(s)=v_{2}^{4}(s)$ по 2) леммы 1.1. Отсюда следует, что базис из 1$)$ леммы 1.1 совпадает с множеством $t_{1}^{4}[L] \backslash\{1\}$ всех неединичных четвертых степеней элементов $L$ (так как если $g^{4} \neq 1$, где $g \in L$, то $\left.g \notin L^{2}\right)$ и, в частности, не зависит от выбора множества представителей $U$. Из 2) леммы 1.1 также вытекает, что отображение $\eta$, заданное формулой

$$
v(y) \eta=v^{4}(s), \quad v(t) \in \mathfrak{R}(F),
$$

определено корректно и отображает взаимно однозначно $\mathfrak{R}(A)$ на $t_{1}^{4}[L]$. В частности, $\mathfrak{R}(A) \backslash\{1\}$ отображается на базис из 1) леммы 1.1.

Из сказанного выше легко вывести следуюшую лемму. 
Лемма $1.2\left(\left[2\right.\right.$, теорема 4.3]). Пусть $W_{1}, W_{2}$ - множества әлементов $\mathfrak{R}(A)$, содержащие 1. Тогда

$$
W_{1} \subseteq W_{2} \Longleftrightarrow\left\langle W_{1} \eta\right\rangle \subseteq\left\langle W_{2} \eta\right\rangle .
$$

В заключение этого параграфа приведем утверждение, которое понадобится нам в дальнейшем.

ЛЕмма 1.3 ([2, лемма 4.6]). Если $\mathfrak{R}$ конечно базируемо, то и $\mathfrak{R}$ конечно базируемо.

\section{§2. Некоторые цепи вербальных подгрупп и подмножеств}

В [6, п. 11.2] описан стандартный процесс построения алгебраической системы по ее порождающим и определяюшим соотношениям в произвольном квазимногообразии $\mathfrak{Q}$. В частности, при пустом множестве определяющих соотношений получается $\mathfrak{Q}$-свободная группа, которая также является var $\mathfrak{Q}$-свободной группой. Нам потребуется следующий вариант этого процесса.

Обозначим через $\mathfrak{U}$ некоторое многообразие групп, а через $\mathfrak{Q}$ - квазимногообразие, заданное в $\mathfrak{U}$ тождеством $v_{0}(t)=1$ и квазитождеством $v_{1}(t)=1 \longrightarrow v_{2}(t)=1$ при некоторых $v_{0}(t), v_{1}(t), v_{2}(t) \in F$. Пусть также $G-\mathfrak{U}$-свободная группа счетного ранга, а $\mathfrak{V}=\operatorname{var} \mathfrak{Q}$. Построим с помощью индукции по $i \in \mathbb{N}_{0}$ следующее семейство подгрупп $G$ :

$$
\begin{aligned}
V_{0} & =v_{0}(G), \\
V_{i+1} & =\left\langle V_{i} \cup\left\{v_{2}(g): g \in G^{*}, v_{1}(g) \in V_{i}\right\}\right\rangle, \quad i \in \mathbb{N}_{0} .
\end{aligned}
$$

Очевидно, что все $V_{i}$ - вполне характеристические подгруппы $G$ и

$$
V_{0} \subseteq V_{1} \subseteq \cdots,
$$

поэтому $\bigcup_{i=0}^{\infty} V_{i}$ - вполне характеристическая (равносильно, вербальная) подгруппа $G$; мы обозначим ее через $V$.

Лемма 2.1. Справедливо следующее: $V=\mathfrak{V}(G)$.

ДокАЗАТЕЛЬСТво. Покажем индукцией по $i \in \mathbb{N}_{0}$, что $V_{i} \subseteq \mathfrak{V}(G)$. При $i=0$ это следует из того, что $\mathfrak{Q} \models v_{0}(t)=1$ и, следовательно, $\mathfrak{V} \models v_{0}(t)=1$. Пусть теперь $z_{1}, z_{2}, \ldots$ - некоторая система свободных порождаюших $G$. Заметим, что для произвольной последовательности $f(t)=f_{1}(t), f_{2}(t), \ldots \in F^{*}$

$$
\begin{aligned}
v_{1}(f(z)) \in \mathfrak{V}(G) & \Longleftrightarrow \mathfrak{V}=v_{1}(f(t))=1 \\
& \Longleftrightarrow \mathfrak{Q}=v_{1}(f(t))=1 \\
& \Longleftrightarrow \mathfrak{Q}=v_{2}(f(t))=1 \\
& \quad\left(\text { так как } \mathfrak{Q}=\left(v_{1}(t)=1 \longrightarrow v_{2}(t)=1\right)\right) \\
& \Longleftrightarrow \mathfrak{V}=v_{2}(f(t))=1 \Longleftrightarrow v_{2}(f(z)) \in \mathfrak{V}(G)
\end{aligned}
$$

(здесь $f(z)$ обозначает последовательность $\left.f_{1}(z), f_{2}(z), \ldots \in G^{*}\right)$. Из этого легко вытекает, что если $V_{i} \subseteq \mathfrak{V}(G)$, то $V_{i+1} \subseteq \mathfrak{V}(G)$ для всех $i \in \mathbb{N}_{0}$. Таким образом, $V_{i} \subseteq \mathfrak{V}(G)$ для всех $i \in \mathbb{N}_{0}$ и, следовательно, $V \subseteq \mathfrak{V}(G)$. 
Для доказательства обратного включения заметим, что $G / V \in \mathfrak{U}, G / V \models$ $v_{0}(t)=1$ (так как $\left.v_{0}(G)=V_{0} \subseteq V\right)$ и $G / V \models\left(v_{1}(t)=1 \longrightarrow v_{2}(t)=1\right)$ (ввиду того, что если $v_{1}(g) \in V$ для некоторой последовательности $g \in G^{*}$, то $v_{1}(g) \in V_{i}$ для некоторого $i \in \mathbb{N}_{0}$ и, следовательно, $\left.v_{2}(g) \in V_{i+1} \subseteq V\right)$. Это значит, что $G / V \in \mathfrak{Q} \subseteq \mathfrak{V}$, поэтому $\mathfrak{V}(G) \subseteq V$. Лемма доказана.

Пусть теперь $w_{0}(t), w_{1}(t), w_{2}(t) \in \mathfrak{R}(F)$. Положим $\mathfrak{U}=\mathfrak{L} \mathfrak{R}, G=S, v_{j}(t)=$ $w_{j}^{4}(t)$ для $j=0,1,2$ и построим подгруппы $V_{i}, i \in \mathbb{N}_{0}$, согласно правилам (1). Определим индукцией по $i \in \mathbb{N}_{0}$ также следующие подмножества $W_{i}$ группы $A$ :

$$
\begin{aligned}
W_{0} & =w_{0}[A], \\
W_{i+1} & =W_{i} \cup\left\{w_{2}(a): a \in A^{*}, w_{1}(a) \in W_{i}\right\}, \quad i \in \mathbb{N}_{0} .
\end{aligned}
$$

Очевидно, что $1 \in W_{i} \subseteq \mathfrak{R}(A), W_{i} \varphi \subseteq W_{i}$ для любого $i \in \mathbb{N}_{0}$ и любого эндоморфизма $\varphi$ группы $A$ и

$$
W_{0} \subseteq W_{1} \subseteq \cdots .
$$

Лемма 2.2. Справедливо следующее: $V_{i}=\left\langle W_{i} \eta\right\rangle$ для всех $i \in \mathbb{N}_{0}$.

ДокАЗАТЕЛЬСТво. Индукция по $i$. При $i=0$ утверждение леммы вытекает непосредственно из определений. Пусть теперь $V_{i}=\left\langle W_{i} \eta\right\rangle$, тогда

$$
\begin{aligned}
V_{i+1} & =\left\langle V_{i} \cup\left\{v_{2}(g): g \in S^{*}, v_{1}(g) \in V_{i}\right\}\right\rangle \\
& =\left\langle V_{i} \cup\left\{w_{2}^{4}(g): g \in S^{*}, w_{1}^{4}(g) \in V_{i}\right\}\right\rangle \\
& =\left\langle V_{i} \cup\left\{w_{2}^{4}(g): g \in S^{*},\left\langle 1, w_{1}^{4}(g)\right\rangle \subseteq V_{i}\right\}\right\rangle \\
& =\left\langle\left\langle W_{i} \eta\right\rangle \cup\left\{w_{2}(a) \eta: a \in A^{*},\left\langle\left\{1, w_{1}(a)\right\} \eta \backslash \subseteq\left\langle W_{i} \eta\right\rangle\right\}\right\rangle\right. \\
& =\left\langle\left\langle W_{i} \eta\right\rangle \cup\left\{w_{2}(a) \eta: a \in A^{*},\left\{1, w_{1}(a)\right\} \subseteq W_{i}\right\}\right\rangle \text { (по лемме 1.2) } \\
& =\left\langle W_{i} \eta \cup\left\{w_{2}(a) \eta: a \in A^{*}, w_{1}(a) \in W_{i}\right\}\right\rangle=\left\langle W_{i+1} \eta\right\rangle .
\end{aligned}
$$

Лемма доказана.

\section{§3. Определение и свойства некоторых слов}

Мы начнем с одного замечания о базисных коммутаторах (для конечного алфавита их определение можно найти, например, в [5, определение 31.51$]$ ).

ЛЕмма 3.1. Пусть а и $b$ - базиснье коммутаторы в одном и том же алфавите, причем а имеет вес $k$, a $b$ - вес $l$. Тогда если $k>l$ u $k / 2<l$, то $[a, b]-$ базисный коммутатор $($ веса $k+l)$.

ДокАЗАТЕЛЬСТВо. Ясно, что $a>b$, так как $k>l$. Если $a=\left[a_{1}, a_{2}\right]$, где $a_{1}$ и $a_{2}$ - базисные коммутаторы весов $k_{1}$ и $k_{2}$, соответственно, то $k_{1} \geqslant k_{2}$ (иначе $a_{1}<a_{2}$ и $a$ - не базисный коммутатор). Поэтому $k_{2} \leqslant\left(k_{1}+k_{2}\right) / 2=k / 2<l$, следовательно, $a_{2}<b$. Таким образом, $[a, b]-$ базисньй коммутатор. Лемма доказана.

Нетрудно видеть, что для четного числа с существуют $k_{1}, k_{2} \in \mathbb{Z}$ такие, что

$$
\begin{gathered}
3 \leqslant k_{i} \leqslant c-3, \quad k_{1} \neq k_{2}, \\
k_{i}>l_{i}, \quad \frac{k_{i}}{2}<l_{i}, \\
\text { где } \quad l_{i}=c-k_{i}, \quad i=1,2,
\end{gathered}
$$


если и только если $c \geqslant 14$. Зафиксируем некоторое четное число $c \geqslant 14$ и положим $\mathfrak{R}=\mathfrak{N}_{c}$ в обозначениях, введенных в $\S 1$. Выберем также некоторые $k_{1}, k_{2} \in \mathbb{Z}$, удовлетворяющие условиям $(5)$, и произвольное число $m \in \mathbb{Z} \backslash\{-1,0,1\}$.

Определим следующие слова из $F$ :

$$
\begin{aligned}
& f_{1}(t)=\left[\left[t_{2},\left(k_{1}-2\right) t_{1}, t_{3}\right],\left[t_{2},\left(l_{1}-2\right) t_{1}, t_{3}\right]\right] \\
& f_{2}(t)=\left[\left[t_{2},\left(k_{2}-1\right) t_{1}\right],\left[t_{2},\left(l_{2}-1\right) t_{1}\right]\right] \\
& u_{1}(t)=f_{1}\left(t_{1}, t_{2}, t_{3}\right) f_{2}(t) \\
& u_{2}(t)=f_{1}\left(t_{1}, t_{2}, t_{3}^{m}\right) f_{2}(t)=u_{1}\left(t_{1}, t_{2}, t_{3}^{m}\right) \\
& u_{0}(t)=f_{1}\left(t_{1}, t_{2}, t_{1}\right) f_{2}(t)=u_{1}\left(t_{1}, t_{2}, t_{1}\right)
\end{aligned}
$$

где $l_{1}, l_{2}$ определены в $(5)$.

ЛЕмМа 3.2. Если $r_{1} r_{2}=r_{1}^{\prime} r_{2}^{\prime}$, әде $r_{i}, r_{i}^{\prime} \in f_{i}(R)$, mo $r_{i}=r_{i}^{\prime}$ при $i=1,2$.

ДокаЗАТЕЛЬСтво. Пусть $B(j)$ - множество всех базисных коммутаторов от $x_{1}, x_{2}, \ldots$ веса $j$. Легко видеть, что

$$
f_{i}[R] \subseteq\left[R_{\left(k_{i}\right)}, R_{\left(l_{i}\right)}\right]=\left[\left\langle B\left(k_{i}\right)\right\rangle,\left\langle B\left(l_{i}\right)\right\rangle\right] \subseteq\left\langle\left[B\left(k_{i}\right), B\left(l_{i}\right)\right]\right\rangle
$$

значит, $f_{i}(R) \subseteq\left\langle\left[B\left(k_{i}\right), B\left(l_{i}\right)\right]\right\rangle$. Но ввиду условий $(5)$ и леммы $3.1\left[B\left(k_{1}\right), B\left(l_{1}\right)\right]$ и $\left[B\left(k_{2}\right), B\left(l_{2}\right)\right]$ - непересекающиеся подмножества $B(c)$, которое является базисом $R_{(c)}$ как свободной абелевой группы. Из этого непосредственно следует утверждение леммы.

Лемма 3.3. Eсли $f_{2}\left(r_{1}, r_{2}\right)=f_{2}\left(x_{1}, x_{2}\right)^{\varepsilon}$, әде $\varepsilon \in\{-1,1\}$ u $r_{1}, r_{2} \in R$, mo $\varepsilon=1 u r_{1} \equiv x_{1}^{\varepsilon_{1}}\left(\bmod R^{\prime}\right), r_{2} \equiv x_{1}^{\xi} x_{2}^{\varepsilon_{2}}\left(\bmod R^{\prime}\right)$ для некоторых $\varepsilon_{1}, \varepsilon_{2} \in\{-1,1\}$, $\xi \in \mathbb{Z}$.

ДокАЗАтЕльство. Пусть $r_{1} \equiv \prod_{i=1}^{\infty} x_{i}^{\alpha_{i}}\left(\bmod R^{\prime}\right)$ и $r_{2} \equiv \prod_{i=1}^{\infty} x_{i}^{\beta_{i}}\left(\bmod R^{\prime}\right)$, где $\alpha_{i}, \beta_{i} \in \mathbb{Z}$ (здесь $\alpha_{i}=\beta_{i}=0$ для всех достаточно больших $i \in \mathbb{N}_{1}$, поэтому произведения определены). Зафиксируем произвольные $i, j \in \mathbb{N}_{1}$ такие, что $i>j$. Обозначим через $\pi_{i j}$ эндоморфизм $R$, определенный на свободных порождающих $x_{1}, x_{2}, \ldots$ следующим образом:

$$
x_{n} \pi_{i j}= \begin{cases}x_{n}, & \text { если } n \in\{i, j\} \\ 1 & \text { в противном случае. }\end{cases}
$$

Для произвольных $n_{1}, \ldots, n_{c-4} \in\{i, j\}$ положим

$$
b_{n_{1} \ldots n_{c-4}}=\left[\left[x_{i}, x_{j}, x_{n_{1}}, \ldots, x_{n_{k_{2}-2}}\right],\left[x_{i}, x_{j}, x_{n_{k_{2}-1}}, \ldots, x_{n_{c-4}}\right]\right] .
$$

Тогда легко видеть, что

$$
f_{2}\left(r_{1}, r_{2}\right) \pi_{i j}=f_{2}\left(x_{j}^{\alpha_{j}} x_{i}^{\alpha_{i}}, x_{j}^{\beta_{j}} x_{i}^{\beta_{i}}\right)=\prod_{n_{1}, \ldots, n_{c-4} \in\{i, j\}} b_{n_{1} \ldots n_{c-4}}^{\gamma_{i_{1} \alpha_{n_{1}} \cdots \alpha_{n_{c-4}}}^{2}}
$$


где $\gamma_{i j}=\alpha_{j} \beta_{i}-\alpha_{i} \beta_{j}$. Из этого ввиду равенства $f_{2}\left(r_{1}, r_{2}\right) \pi_{i j}=f_{2}\left(x_{1}, x_{2}\right)^{\varepsilon} \pi_{i j}$ следует, что

$$
b_{i i \ldots i}^{\gamma_{i j}^{2} \alpha_{i}^{c-4}} b_{j j \ldots j}^{\gamma_{i j}^{2} \alpha_{j}^{c-4}} r=\left[\left[x_{2},\left(k_{2}-1\right) x_{1}\right],\left[x_{2},\left(l_{2}-1\right) x_{1}\right]\right]^{\varepsilon} \pi_{i j},
$$

где $r$-произведение степеней базисных коммутаторов от $x_{1}, x_{2}, \ldots$ веса $c$, отличных от $b_{i i \ldots i}$ и $b_{j j \ldots j}$ (очевидно, что $b_{i i \ldots i}$ и $b_{j j \ldots j}$ - базисные коммутаторы от $x_{1}, x_{2}, \ldots$ веса $\left.c\right)$. Поэтому

$$
\begin{aligned}
& \gamma_{i j}^{2} \alpha_{i}^{c-4}=0, \\
& \gamma_{i j}^{2} \alpha_{j}^{c-4}= \begin{cases}\varepsilon, & \text { если } i=2 \text { и } j=1, \\
0 & \text { в противном случае }\end{cases}
\end{aligned}
$$

ввиду независимости базисных коммутаторов от $x_{1}, x_{2}, \ldots$ веса $c$. В частности,

1) $\varepsilon=1$ и $\alpha_{1}, \gamma_{21} \in\{-1,1\}$, так как $\left(\gamma_{21} \alpha_{1}^{(c-4) / 2}\right)^{2}=\varepsilon$;

2) $\alpha_{2}=0$, так как $\alpha_{2}^{c-4}=\gamma_{21}^{2} \alpha_{2}^{c-4}=0$;

3) $\beta_{2} \in\{-1,1\}$, так как $\alpha_{1} \beta_{2}=\alpha_{1} \beta_{2}-\alpha_{2} \beta_{1}=\gamma_{21} \in\{-1,1\}$;

4) $\alpha_{i}=0$ при $i \in \mathbb{N}_{3}$, так как $\alpha_{i}^{c-2}=\alpha_{i}^{c-2} \beta_{2}^{2}=\left(\alpha_{2} \beta_{i}-\alpha_{i} \beta_{2}\right)^{2} \alpha_{i}^{c-4}=$ $\gamma_{i 2}^{2} \alpha_{i}^{c-4}=0$

5) $\beta_{i}=0$ при $i \in \mathbb{N}_{3}$, так как $\beta_{i}^{2}=\alpha_{1}^{c-2} \beta_{i}^{2}=\left(\alpha_{1} \beta_{i}-\alpha_{i} \beta_{1}\right)^{2} \alpha_{1}^{c-4}=$ $\gamma_{i 1}^{2} \alpha_{1}^{c-4}=0$

что и требовалось. Лемма доказана.

Лемма 3.4. Если $f_{1}\left(r_{1}, r_{2}, r_{3}\right)=f_{1}\left(x_{1}, x_{2}, r\right)$, где $r_{1}, r_{2}, r_{3}, r \in R$, причем $r_{1} \equiv x_{1}^{\varepsilon_{1}}\left(\bmod R^{\prime}\right), r_{2} \equiv x_{1}^{\xi} x_{2}^{\varepsilon_{2}}\left(\bmod R^{\prime}\right)$ для некоторих $\varepsilon_{1}, \varepsilon_{2} \in\{-1,1\}, \xi \in \mathbb{Z}$, mо $r_{3} \equiv r^{\varepsilon_{3}}\left(\bmod R^{\prime}\right)$ при некотором $\varepsilon_{3} \in\{-1,1\}$.

ДОКАЗАТЕЛЬСТВо. Легко видеть, что

$$
f_{1}\left(r_{1}, r_{2}, r_{3}\right)=f_{1}\left(x_{1}^{\varepsilon_{1}}, x_{2}^{\varepsilon_{2}}, r_{3}\right)=f_{1}\left(x_{1}, x_{2}, r_{3}\right)^{\varepsilon_{1}^{c-4} \varepsilon_{2}^{2}}=f_{1}\left(x_{1}, x_{2}, r_{3}\right),
$$

так как $c$ четно. Пусть $r_{3} \equiv \prod_{i=1}^{\infty} x_{i}^{\gamma_{i}}\left(\bmod R^{\prime}\right), r \equiv \prod_{i=1}^{\infty} x_{i}^{\alpha_{i}}\left(\bmod R^{\prime}\right)$, где $\gamma_{i}, \alpha_{i} \in \mathbb{Z}$ (здесь $\gamma_{i}=\alpha_{i}=0$ для всех достаточно больших $i \in \mathbb{N}_{1}$, поэтому произведения определены). Тогда

$$
\begin{aligned}
f_{1}\left(r_{1}, r_{2}, r_{3}\right) & =\prod_{i, j=1}^{\infty}\left[\left[x_{2},\left(k_{1}-2\right) x_{1}, x_{i}\right],\left[x_{2},\left(l_{1}-2\right) x_{1}, x_{j}\right]\right]^{\gamma_{i} \gamma_{j}} \\
f_{1}\left(x_{1}, x_{2}, r\right) & =\prod_{i, j=1}^{\infty}\left[\left[x_{2},\left(k_{1}-2\right) x_{1}, x_{i}\right],\left[x_{2},\left(l_{1}-2\right) x_{1}, x_{j}\right]\right]^{\alpha_{i} \alpha_{j}}
\end{aligned}
$$

где для произвольных $i, j \in \mathbb{N}_{1}\left[\left[x_{2},\left(k_{1}-2\right) x_{1}, x_{i}\right],\left[x_{2},\left(l_{1}-2\right) x_{1}, x_{j}\right]\right]$ является базисным коммутатором веса $c$. Следовательно,

$$
\gamma_{i} \gamma_{j}=\alpha_{i} \alpha_{j} \quad \forall i, j \in \mathbb{N}_{1}
$$

ввиду условия леммы и независимости базисных коммутаторов от $x_{1}, x_{2}, \ldots$ веса $c$.

Пусть $I=\left\{i \in \mathbb{N}_{1}: \alpha_{i} \neq 0\right\}$. Для каждого $i \in I$ положим $\delta_{i}=\gamma_{i} / \alpha_{i}$. Ввиду формулы (7) $\gamma_{i}^{2}=\alpha_{i}^{2}$ для всех $i \in \mathbb{N}_{1}$. Следовательно, если $i \in I$, то $\delta_{i}^{2}=1$ и $\delta_{i} \in\{-1,1\}$, а если $i \in \mathbb{N}_{1} \backslash I$ (т.е. $\left.\alpha_{i}=0\right)$, то $\gamma_{i}=0=\alpha_{i}$. Кроме того, для любых $i, j \in I$ из (7) следует, что $\delta_{i} \delta_{j}=1$, поэтому $\delta_{i}=\delta_{j}$. Таким образом, если $\varepsilon_{3}-$ обшее значение $\delta_{i}$ для всех $i \in I$ (принадлежашее $\{-1,1\}$ ), то $\gamma_{i}=\varepsilon_{3} \alpha_{i}$ для всех $i \in \mathbb{N}_{1}$ и, следовательно, $r_{3} \equiv r^{\varepsilon_{3}}\left(\bmod R^{\prime}\right)$. Лемма доказана. 
Лемма 3.5. Пусть $\varepsilon \in\{-1,1\}$ и $r_{1}, r_{2}, r_{3}, r \in R$. Тогда если $u_{1}\left(r_{1}, r_{2}, r_{3}\right)=$ $u_{1}\left(x_{1}, x_{2}, r\right)^{\varepsilon}$, mo $\varepsilon=1 u$

$$
\begin{gathered}
r_{1} \equiv x_{1}^{\varepsilon_{1}}\left(\bmod R^{\prime}\right), \quad r_{2} \equiv x_{1}^{\xi} x_{2}^{\varepsilon_{2}}\left(\bmod R^{\prime}\right), \quad r_{3} \equiv r^{\varepsilon_{3}}\left(\bmod R^{\prime}\right) \\
\text { для некоторьх } \varepsilon_{1}, \varepsilon_{2}, \varepsilon_{3} \in\{-1,1\}, \quad \xi \in \mathbb{Z} .
\end{gathered}
$$

Обратно, если выполнены условия (8), то $u_{1}\left(r_{1}, r_{2}, r_{3}\right)=u_{1}\left(x_{1}, x_{2}, r\right)$.

ДокАЗАТЕЛЬСтво. Пусть сначала $u_{1}\left(r_{1}, r_{2}, r_{3}\right)=u_{1}\left(x_{1}, x_{2}, r\right)^{\varepsilon}$, т.е.

$$
f_{1}\left(r_{1}, r_{2}, r_{3}\right) f_{2}\left(r_{1}, r_{2}\right)=f_{1}\left(x_{1}, x_{2}, r\right)^{\varepsilon} f_{2}\left(x_{1}, x_{2}\right)^{\varepsilon} .
$$

Ввиду леммы 3.2

$$
\begin{aligned}
f_{1}\left(r_{1}, r_{2}, r_{3}\right) & =f_{1}\left(x_{1}, x_{2}, r\right)^{\varepsilon}, \\
f_{2}\left(r_{1}, r_{2}\right) & =f_{2}\left(x_{1}, x_{2}\right)^{\varepsilon} .
\end{aligned}
$$

По лемме 3.3 из формулы $(10)$ следует, что $\varepsilon=1$ и $r_{1} \equiv x_{1}^{\varepsilon_{1}}\left(\bmod R^{\prime}\right), r_{2} \equiv x_{1}^{\xi} x_{2}^{\varepsilon_{2}}$ $\left(\bmod R^{\prime}\right)$ для некоторых $\varepsilon_{1}, \varepsilon_{2} \in\{-1,1\}, \xi \in \mathbb{Z}$. Из этого и из формулы $(9)$ согласно лемме 3.4 вытекает, что $r_{3} \equiv r^{\varepsilon_{3}}\left(\bmod R^{\prime}\right)$ при некотором $\varepsilon_{3} \in\{-1,1\}$. Таким образом, условия (8) выполнены.

Обратно, если вьполнены условия (8), то

$$
\begin{aligned}
f_{1}\left(r_{1}, r_{2}, r_{3}\right) & =f_{1}\left(x_{1}^{\varepsilon_{1}}, x_{2}^{\varepsilon_{2}}, r^{\varepsilon_{3}}\right)=f_{1}\left(x_{1}, x_{2}, r\right)^{\varepsilon_{1}^{c-4}} \varepsilon_{2}^{2} \varepsilon_{3}^{2}=f_{1}\left(x_{1}, x_{2}, r\right), \\
f_{2}\left(r_{1}, r_{2}\right) & =f_{2}\left(x_{1}^{\varepsilon_{1}}, x_{2}^{\varepsilon_{2}}\right)=f_{2}\left(x_{1}, x_{2}\right)^{\varepsilon_{1}^{c-2}} \varepsilon_{2}^{2}=f_{2}\left(x_{1}, x_{2}\right),
\end{aligned}
$$

так как $c$ четно. Поэтому $u_{1}\left(r_{1}, r_{2}, r_{3}\right)=u_{1}\left(x_{1}, x_{2}, r\right)$. Лемма доказана.

\section{§4. Доказательство основного результата}

Пусть, как и в $\S 3, c-$ произвольное четное число, не меньшее $14, k_{1}, k_{2}$ - некоторые целые числа, удовлетворяющие условиям (5), $m$ - произвольное целое число, не принадлежащее $\{-1,0,1\}$, a $\mathfrak{R}=\mathfrak{N}_{c}$. Определим следуюшие слова из $F$ :

$$
\begin{aligned}
f_{0}(t) & =\left[t_{2}, c t_{1}\right], \\
w_{j}(t) & =\left[f_{0}(t), u_{j}(t)\right], \quad j=0,1,2,
\end{aligned}
$$

где $u_{j}(t)$ - слова из (6). Обозначим через $\mathfrak{Q}=\mathfrak{Q}\left(c, k_{1}, k_{2}, m\right)$ квазимногообразие, определенное в многообразии $\mathfrak{L} \mathfrak{R}=\mathfrak{A}_{2}^{3} \mathfrak{N}_{c} \cap\left[\mathfrak{A}_{2}^{2} \mathfrak{N}_{c}, \mathfrak{N}_{c}\right]$ тождеством $w_{0}^{4}(t)=1$ и квазитождеством $w_{1}^{4}(t)=1 \longrightarrow w_{2}^{4}(t)=1$, как в $\S 2$. Цель этого параграфа показать, что квазимногообразия $\mathfrak{Q}\left(c, k_{1}, k_{2}, m\right)$ удовлетворяют условию теоремы 1. Очевидно, что $\mathfrak{Q}$ разрешимо (так как $\mathfrak{L} \mathfrak{R}$ разрешимо), причем при $c=14$ ступень разрешимости $\mathfrak{Q}$ не превосходит 7 (ввиду того, что $\mathfrak{R} \subseteq \mathfrak{A}^{4}$ и, следовательно, $\left.\mathfrak{L} \mathfrak{R} \subseteq \mathfrak{A}^{7}\right)$. Кроме того, квазимногообразие $\mathfrak{Q}$ конечно базируемо, так как многообразие $\mathfrak{L} \mathfrak{R}$ конечно базируемо по лемме 1.3 .

Докажем теперь, что $\mathfrak{V}=\operatorname{var} \mathfrak{Q}$ не конечно базируемо. Положим $\mathfrak{U}=\mathfrak{L} \mathfrak{R}$, $G=S, v_{j}(t)=w_{j}^{4}(t)$ при $j=0,1,2$ и построим цепь (2) вербальных подгрупп $V_{i}$ 
группы $S$ согласно правилам (1). По лемме 2.1 объединение цепи (2) совпадает с $\mathfrak{V}(S)$, поэтому для доказательства отсутствия конечного базиса тождеств $\mathfrak{V}$ достаточно показать, что цепь (2) не стабилизируется ни на каком шаге. В свою очередь, ввиду лемм 2.2 и 1.2 последнее условие равносильно тому, что цепा (4) подмножеств $W_{i}$ группы $A$, построенная по правилам (3), не стабилизируется ни на каком шаге. Таким образом, отсутствие конечного базиса тождеств $\mathfrak{V}$ будет доказано, если мы покажем, что элемент

$$
d_{i}=w_{1}\left(y_{1}, y_{2}, y_{1}^{m^{i}}\right)=\left[f_{0}(y), u_{1}\left(y_{1}, y_{2}, y_{1}^{m^{i}}\right)\right], \quad i \in \mathbb{N}_{0}
$$

групшы $A$ принадлежит $W_{i}$, но не принадлежит $W_{i-1}$ для всех $i \in \mathbb{N}_{1}$. Это будет сделано в леммах 4.3 и 4.4 .

Пусть $H$ wr $R$ - дискретное сплетение групп $H$ и $R$. Мы считаем базу $B$ этого сплетения мультипликативно записываемым свободным правым $\mathbb{Z}_{2} R$-модулем с базисом $h_{1}, h_{2}, \ldots$. Согласно известной теореме Магнуса-Шмелькина о вложении (см., например, [5, теорема 22.48]) гомоморфизм $A \rightarrow H$ wr $R$, отображающий $y_{i}$ в $x_{i} h_{i}$ для всех $i \in \mathbb{N}_{1}$, инъективен; мы обозначим этот гомоморфизм через $\mu$. Нам потребуется также следующая формула, проверяемая непосредственно:

$$
\left[r_{1} \alpha_{1}, r_{2} \alpha_{2}\right]=\left[r_{1}, r_{2}\right] \alpha_{1}^{r_{2}-\left[r_{1}, r_{2}\right]} \alpha_{2}^{1-r_{1}^{r_{2}}}
$$

где $r_{1}, r_{2} \in R$, а $\alpha_{1}, \alpha_{2} \in B$. В частности, для любых $a \in \mathfrak{R}(A), g(t) \in F$

$$
[a, g(y)] \mu=[a \mu, g(x) \alpha]=a \mu^{g(x)-1}
$$

так как $a \mu \in B$ (здесь $\alpha-$ некоторьй элемент $B)$.

Мы будем называть элемент $r \in R$ истинной степенъю в $R$, если $r=r_{0}^{e}$ для некоторых $r_{0} \in R, e \in \mathbb{Z} \backslash\{-1,0,1\}$.

Аналог следуюшей леммы был доказан в [4, лемма 1.9].

ЛЕмма 4.1. Пусть $\left[a, g_{1}(y)\right]=\left[f_{0}(y), g_{2}(y)\right]$, где $a \in \mathfrak{R}(A)=A_{(c+1)}, g_{1}(t), g_{2}(t)$ $\in F_{(c)}$, причем $g_{2}(x)$ не является истинной степенью в $R$. Тогда $g_{1}(x)=g_{2}(x)^{\varepsilon}$ при некотором $\varepsilon \in\{-1,1\}$.

ДоКАЗАТЕЛЬСТвО. Используя формулы (11) и (12), вычислим координаты при $h_{2}$ элементов $\left[a, g_{1}(y)\right] \mu$ и $\left[f_{0}(y), g_{2}(y)\right] \mu$. Приравняв эти координаты, получим, что

$$
\begin{aligned}
\alpha\left(g_{1}(x)-1\right) & =\left(x_{1}-\left[x_{2}, x_{1}\right]\right)\left(x_{1}-\left[x_{2}, 2 x_{1}\right]\right) \cdots\left(x_{1}-\left[x_{2}, c x_{1}\right]\right)\left(g_{2}(x)-1\right) \\
& =\sum_{r_{1}, \ldots, r_{c}} r_{1} \cdots r_{c}\left(g_{2}(x)-1\right)
\end{aligned}
$$

где $\alpha \in \mathbb{Z}_{2} R$ - координата при $h_{2}$ элемента $а \mu$ и суммирование производится по всевозможным упорядоченным наборам $\left(r_{1}, \ldots, r_{c}\right)$, в которых $r_{i}$ независимо друг от друга пробегают множества $\left\{x_{1},\left[x_{2}, i x_{1}\right]\right\}$. Очевидно, что

$$
\begin{aligned}
\left(r_{1}, \ldots, r_{c}\right) \neq\left(x_{1}, x_{1}, \ldots, x_{1}\right) & \Longrightarrow x_{1}^{c} \not \equiv r_{1} \cdots r_{c}\left(\bmod R^{\prime}\right) \\
& \Longrightarrow x_{1}^{c} \not \equiv r_{1} \cdots r_{c}\left(\bmod R_{(c)}\right)
\end{aligned}
$$


Выберем некоторое множество $M$ представителей всех смежных классов $R$ по $R_{(c)}$, содержашее $x_{1}^{c}$. Тогда $M$ является базисом $\mathbb{Z}_{2} R$ как свободного правого $\mathbb{Z}_{2} R_{(c)}$-модуля. Из (13) следует, что в этом базисе координата при $x_{1}^{c}$ элемента $\sum_{r_{1}, \ldots, r_{c}} r_{1} \cdots r_{c}\left(g_{2}(x)-1\right)$ равна $g_{2}(x)-1$. Приравняв эту координату к координате при $x_{1}^{c}$ элемента $\alpha\left(g_{1}(x)-1\right)$, получим, что $g_{2}(x)-1$ делится на $g_{1}(x)-1$ в кольце $\mathbb{Z}_{2} R_{(c)}$. Следовательно, $g_{2}(x)=g_{1}(x)^{\varepsilon}$ для некоторого $\varepsilon \in \mathbb{Z}$ (переходим от $R_{(c)}$ к $\left.R_{(c)} /\left\langle g_{1}(x)\right\rangle\right)$, причем $\varepsilon \in\{-1,1\}$ ввиду того, что $g_{2}(x)$ не является истинной степенью в $R$. Поэтому $g_{1}(x)=g_{1}(x)^{\varepsilon^{2}}=g_{2}(x)^{\varepsilon}$. Лемма доказана.

Лемма 4.2. Справедливо следующее: $d_{i} \notin W_{0}$ для любого $i \in \mathbb{N}_{1}$.

ДокАЗАТЕЛЬСтво. Пусть, напротив,

$$
\begin{aligned}
d_{i} & =\left[f_{0}(y), u_{1}\left(y_{1}, y_{2}, y_{1}^{m^{i}}\right)\right] \\
& =\left[f_{0}\left(a_{1}(y), a_{2}(y)\right), u_{0}\left(a_{1}(y), a_{2}(y)\right)\right] \\
& =\left[f_{0}\left(a_{1}(y), a_{2}(y)\right), u_{1}\left(a_{1}(y), a_{2}(y), a_{1}(y)\right)\right]
\end{aligned}
$$

при некоторых $i \in \mathbb{N}_{1}, a_{1}(t), a_{2}(t) \in F$. Очевидно, что $u_{1}\left(x_{1}, x_{2}, x_{1}^{m^{i}}\right)$ не является истинной степенью в $R$, так как $u_{1}\left(x_{1}, x_{2}, x_{1}^{m^{i}}\right)=f_{1}\left(x_{1}, x_{2}, x_{1}\right)^{m^{2 i}} f_{2}(x)$, где $f_{1}\left(x_{1}, x_{2}, x_{1}\right)$ и $f_{2}(x)$ - различные базисные коммутаторы от $x_{1}, x_{2} \ldots$ веса $c$. По лемме 4.1 из (14) следует, что $u_{1}\left(a_{1}(x), a_{2}(x), a_{1}(x)\right)=u_{1}\left(x_{1}, x_{2}, x_{1}^{m^{i}}\right) \varepsilon$ при некотором $\varepsilon \in\{-1,1\}$. Согласно лемме $3.5 x_{1}^{m^{i} \varepsilon_{3}} \equiv a_{1}(x) \equiv x_{1}^{\varepsilon_{1}}\left(\bmod R^{\prime}\right)$ при некоторых $\varepsilon_{1}, \varepsilon_{3} \in\{-1,1\}$. Но тогда $m^{i} \varepsilon_{3}=\varepsilon_{1}$ и, следовательно, $m \in\{-1,1\}$ (так как $i \geqslant 1$ ), что противоречит выбору $m$. Лемма доказана.

Лемма 4.3. Справедливо следующее: $d_{i} \in W_{i}$ для любого $i \in \mathbb{N}_{0}$.

ДОКАЗАТЕЛЬСТВО. Индукция по $i$. При $i=0 d_{0}=w_{0}(y) \in w_{0}[A]=W_{0}$, а если $d_{i}=w_{1}\left(y_{1}, y_{2}, y_{1}^{m^{i}}\right) \in W_{i}$, то $d_{i+1}=w_{1}\left(y_{1}, y_{2}, y_{1}^{m^{i+1}}\right)=w_{2}\left(y_{1}, y_{2}, y_{1}^{m^{i}}\right) \in W_{i+1}$ по определению $W_{i+1}$. Лемма доказана.

Лемма 4.4. Справедливо следующее: $d_{i} \notin W_{i-1}$ для любого $i \in \mathbb{N}_{1}$.

ДокАЗАТЕЛЬСТво. Предположим, что сушествуют $j \in \mathbb{N}_{1}$, для которых $d_{j} \in$ $W_{j-1}$. Пусть $i=\min \left\{j \in \mathbb{N}_{1}: d_{j} \in W_{j-1}\right\}$ и $n=\min \left\{j \in \mathbb{N}_{0}: d_{i} \in W_{j}\right\}$. Из леммы 4.2 вытекает, что $i \geqslant 2$ и $n \geqslant 1$. Кроме того, $n \leqslant i-1$ в силу выбора $i$ и $n$. По определению $W_{n} d_{i}=w_{1}\left(y_{1}, y_{2}, y_{1}^{m^{i}}\right)=w_{2}\left(a_{1}(y), a_{2}(y), a_{3}(y)\right)$ и $w_{1}\left(a_{1}(y), a_{2}(y), a_{3}(y)\right) \in W_{n-1}$ для некоторых $a_{1}(t), a_{2}(t), a_{3}(t) \in F$, так как $d_{i} \notin W_{n-1}$. Первое из этих условий означает, что

$$
\left[f_{0}(y), u_{1}\left(y_{1}, y_{2}, y_{1}^{m^{i}}\right)\right]=\left[f_{0}\left(a_{1}(y), a_{2}(y)\right), u_{2}\left(a_{1}(y), a_{2}(y), a_{3}(y)\right)\right] .
$$

Как мы уже видели при доказательстве леммы $4.2, u_{1}\left(x_{1}, x_{2}, x_{1}^{m^{i}}\right)$ не является истинной степенью в $R$. По лемме 4.1 из (15) следует, что $u_{2}\left(a_{1}(x), a_{2}(x), a_{3}(x)\right)=$ $u_{1}\left(a_{1}(x), a_{2}(x), a_{3}^{m}(x)\right)=u_{1}\left(x_{1}, x_{2}, x_{1}^{m^{i}}\right)^{\varepsilon}$ для некоторого $\varepsilon \in\{-1,1\}$. Согласно лемме $3.5 \quad \varepsilon=1$ и

$a_{1}(x) \equiv x_{1}^{\varepsilon_{1}} \quad\left(\bmod R^{\prime}\right), \quad a_{2}(x) \equiv x_{1}^{\xi} x_{2}^{\varepsilon_{2}} \quad\left(\bmod R^{\prime}\right), \quad a_{3}^{m}(x) \equiv x_{1}^{m^{i} \varepsilon_{3}} \quad\left(\bmod R^{\prime}\right)$ 
для некоторых $\varepsilon_{1}, \varepsilon_{2}, \varepsilon_{3} \in\{-1,1\}, \xi \in \mathbb{Z}$. Из последнего сравнения следует, что $a_{3}(x) \equiv x_{1}^{m^{i-1} \varepsilon_{3}}\left(\bmod R^{\prime}\right)$, так как $m \neq 0$. Поэтому $u_{1}\left(a_{1}(x), a_{2}(x), a_{3}(x)\right)=$ $u_{1}\left(x_{1}, x_{2}, x_{1}^{m^{i-1}}\right)$ ввиду леммы 3.5. Применив к равенству (15) гомоморфизм $\mu$ и воспользовавшись формулой (12), получим, что если $r=u_{2}\left(a_{1}(x), a_{2}(x), a_{3}(x)\right)=$ $u_{1}\left(x_{1}, x_{2}, x_{1}^{m^{i}}\right)$, то

$$
f_{0}(y) \mu^{r-1}=f_{0}\left(a_{1}(y), a_{2}(y)\right) \mu^{r-1} .
$$

Из этого равенства следует, что $f_{0}(y) \mu=f_{0}\left(a_{1}(y), a_{2}(y)\right) \mu$, так как $r-1$ не является делителем нуля в кольце $\mathbb{Z}_{2} R$. Используя формулу (12), получаем, что

$$
\begin{aligned}
d_{i-1} \mu & =f_{0}(y) \mu^{u_{1}\left(x_{1}, x_{2}, x_{1}^{m^{i-1}}\right)-1} \\
& =f_{0}\left(a_{1}(y), a_{2}(y)\right) \mu^{u_{1}\left(a_{1}(x), a_{2}(x), a_{3}(x)\right)-1} \\
& =w_{1}\left(a_{1}(y), a_{2}(y), a_{3}(y)\right) \mu .
\end{aligned}
$$

Следовательно, $d_{i-1}=w_{1}\left(a_{1}(y), a_{2}(y), a_{3}(y)\right) \in W_{n-1} \subseteq W_{i-2}$ (напомним, что $\mu$ инъективно), что противоречит выбору $i$. Лемма доказана.

\section{Список литературы}

1. Мазуров В. Д., Хухро Е.И. Нерешенные вопросы теории групп. Коуровская тетрадь. Новосибирск: Изд-во НИИ математико-информационных основ обучения НГУ, 1995.

2. Клейман Ю. Г. О некоторых вопросах теории многообразий групп // Изв. АН СССР. Сер. матем. 1983. Т. 47. №1. С. 37-74.

3. Vaughan-Lee M. R. Uncountably many varieties of groups // Bull. London Math. Soc. 1970. V. 2. Pt. 3. №6. P. 280-286.

4. Клейман Ю. Г. О тождествах в группах // Труды ММО. 1982. Т. 44. С. 62-108.

5. Нейман Х. Многообразия групп. М.: Мир, 1969.

6. Мальцев А.И. Алгебраические системы. М.: Наука, 1970.

Московский государственнњй

Поступила в редакцию

университет им. М.В. Ломоносова

09.06.1997 